\title{
Room-Temperature Reduction of Sulfur Hexafluoride with Metal Phosphides
}

\author{
Blake S. N. Huchenski, Alexander W. H. Speed*
}

Upon treatment with sulfur hexafluoride, alkali metal diphenyl or dicyclohexyl phosphides are oxidized within seconds to tetraphenyl or tetracyclohexyl diphosphines. In a hindered example, di-tert-butylphosphide, fluorophosphine intermediates are detected. This is the first reported reaction of sulfur hexafluoride with metal phosphides, and a rare example of reactivity of sulfur hexafluoride at ambient temperature.

Sulfur hexafluoride $\left(\mathrm{SF}_{6}\right)$ is an industrially used gas, with many applications depending on its density, high dielectric constant, and relative inertness. An unfortunate side effect of this relative inertness is that $\mathrm{SF}_{6}$ released into the atmosphere has a lifetime of thousands of years, resulting in it having the highest global warming potential ( 23500 relative to $\mathrm{CO}_{2}$ over a 100 year time-frame) of any commonly used industrial gas. ${ }^{1} \mathrm{SF}_{6}$ is used most extensively in electrical equipment as an insulator and arc-quencher. ${ }^{2}$ This application, involving the use of many thousands of tonnes of $\mathrm{SF}_{6}$, mandates controls on its use and release. ${ }^{3}$ Because of the high global warming potential of $\mathrm{SF}_{6}$, and its common use, chemistry to decompose surplus $\mathrm{SF}_{6}$ remains a sought after goal. The stability of $\mathrm{SF}_{6}$ arises from a kinetic barrier to decomposition, rather than high thermodynamic stability. The central sulfur is well shielded from nucleophilic attack by the fluorides. Harsh conditions, such as exposure to Lewis acids at high temperature and pressure, or electric-arc plasmas have been used to decompose sulfur hexafluoride. ${ }^{4}$ More recently, several relatively mild solution-phase strategies resulting in stoichiometric reaction of $\mathrm{SF}_{6}$ with a reductant have been disclosed. Low valent early metal complexes, and electron rich nickel complexes are fluorinated by $\mathrm{SF}_{6} .{ }^{5} \mathrm{SF}_{6}$ is reduced by solvated electrons in ammonia solutions. ${ }^{6}$

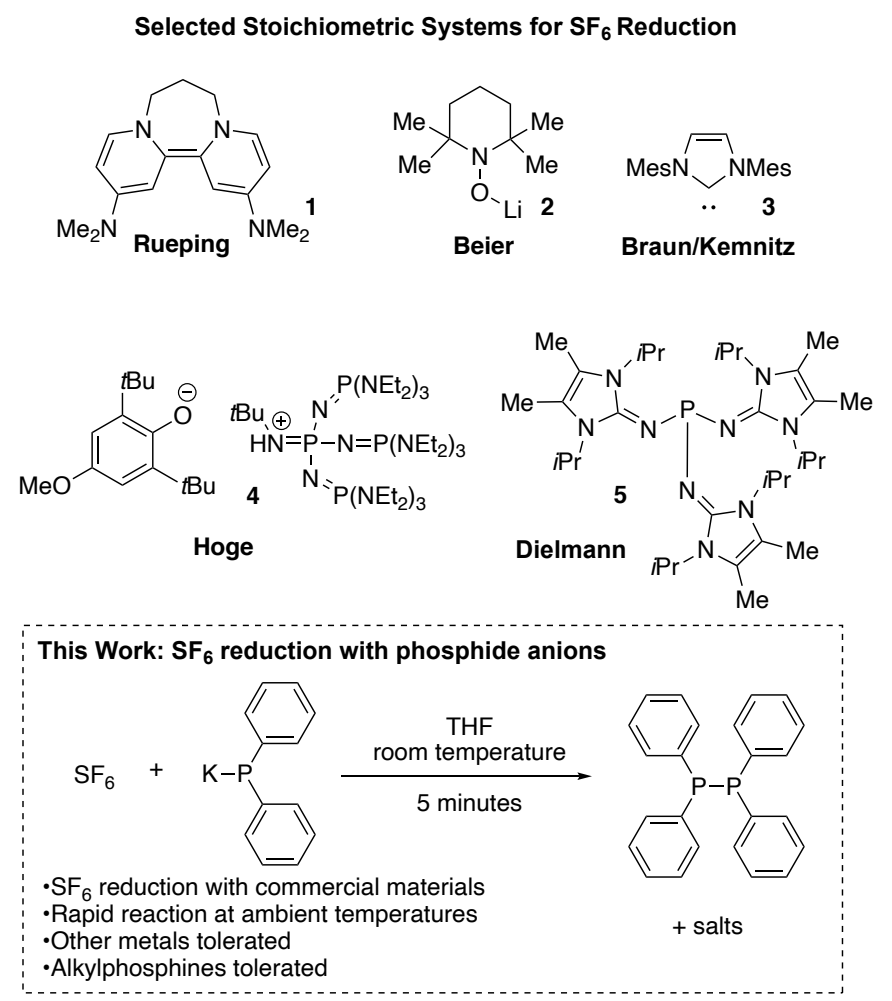

Scheme 1. Selected stoichiometric systems for $\mathrm{SF}_{6}$ degradation 
Select examples of main-group molecules that react with $\mathrm{SF}_{6}$ are shown in Scheme 1. Rueping showed reduction of $\mathrm{SF}_{6}$ in solutions using Murphy's electron-donor reagent $1 .^{7}$ Beier showed reduction of $\mathrm{SF}_{6}$ using the lithium salt of the TEMPO anion 2. ${ }^{8}$ Braun and Kemnitz showed fluorination of $\mathrm{N}$-heterocyclic carbene $\mathbf{3}$ by $\mathrm{SF}_{6}$ under UV irradiation. $^{9}$ During the course of this work, Hoge showed reduction of $\mathrm{SF}_{6}$ with an electron rich phenolate anion/protonated phosphazene pair 4. ${ }^{10}$ Dielmann employed a nucleophilic super-basic phosphine complex for stoichiometric $\mathrm{SF}_{6}$ activation. ${ }^{11}$

Catalytic reactions for functionalization of $\mathrm{SF}_{6}$ have also been reported. Braun and coworkers have reported rhodiumcomplex catalyzed decomposition of $\mathrm{SF}_{6}$ with phosphines as sulfur scavengers and silanes as the terminal reductant. ${ }^{12}$ Several photoredox systems that activate $\mathrm{SF}_{6}$ have been reported. $\mathrm{SF}_{6}$ has recently been used by Jamison as a fluorinating reagent for allylic alcohols employing ruthenium and iridium complex photosensitizers. ${ }^{13}$ Nagorny showed fluorination of carbohydrates using benzophenone-based photosensitizers. ${ }^{14}$ Wagenknecht showed phenothiazinederived photosensitizers have allowed addition of $\mathrm{SF}_{5}$ radicals derived from $\mathrm{SF}_{6}$ to 1,1-diphenylethylene. ${ }^{15}$

Despite the relative simplicity of the systems shown in Scheme 1, access to most of these $\mathrm{SF}_{6}$-reactive examples require multi-step synthesis or relatively harsh conditions, so there is still much room for discovery of simple $\mathrm{SF}_{6}$-reactive systems. Inspired by Dielmann's use of strongly basic phosphines for $\mathrm{SF}_{6}$ functionalization, we explored if simply employing the conjugate base (ie metalation) of a secondary phosphine could result in an increase in basicity sufficient to afford reactivity with $\mathrm{SF}_{6}$. Phosphides have been used in other small molecule activations, notably alkali di-tertbutylphosphides were recently shown by Stephan to activate dihydrogen. ${ }^{16}$

Gratifyingly, metal phosphides proved to be highly reactive with $\mathrm{SF}_{6}$. Bubbling $\mathrm{SF}_{6}$ through a commercially available solution of potassium diphenylphosphide $6 \mathbf{a}$ in THF resulted in disappearance of the deep orange colour within seconds (Equation 1, Scheme 2). ${ }^{17}$ Analysis of the reaction mixture by ${ }^{31} \mathrm{P}$ NMR spectroscopy revealed complete consumption of phosphide, and the formation of tetraphenyldiphosphine 7a. The reaction was relatively clean. Analysis of the reaction mixture by ${ }^{19} \mathrm{~F}$ NMR spectroscopy revealed no sharp peaks associated with new products, however excess $\mathrm{SF}_{6}$ is observed in solution. Despite this, we were able to account for the fate of the fluoride. The volatiles were removed, and the resulting solids were extracted with pentane and diethyl ether (toluene), resulting in removal of 7a. The remaining solids were dissolved in water and ${ }^{19} \mathrm{~F}$ NMR spectroscopy showed a strong signal attributed to KF. To detect sulfide, exposure of the aqueous solution to acidic $\mathrm{N}, \mathrm{N}$-dimethylphenylenediamine $\mathbf{9}$ and ferric chloride resulted in the formation of the blue colour characteristic of methylene blue 10, which is a classic test for sulfide ion (Equation 2 , Scheme 2). ${ }^{18}$ This was not a quantitative test, and materials that were insoluble in both organic solvents and water were noted, which may be elemental sulfur or polysulfides.

The reaction was conducted on a $1 \mathrm{mmol}$ scale with no loss of efficiency, and 7a was isolated from this reaction in $95 \%$ yield, albeit with some inseparable phosphine oxide impurities. A temperature rise was observed during this larger reaction. Metalation of diphenylphosphine 8a with butyllithium in THF followed by introduction of $\mathrm{SF}_{6}$ also resulted in formation of 7a showing that other metals could be used (Equation 3, Scheme 2). ${ }^{19}$

The sulfide detection test also gave a strong blue colour with material from this reaction. Dicyclohexylphosphine $\mathbf{8 b}$ was also deprotonated by butyllithium and upon treatment with $\mathrm{SF}_{6}$, a similar dimerization to $\mathbf{7 b}$ was observed, indicating the reaction was not limited to aryl phosphides (Equation 4 Scheme 2). ${ }^{20}$ 


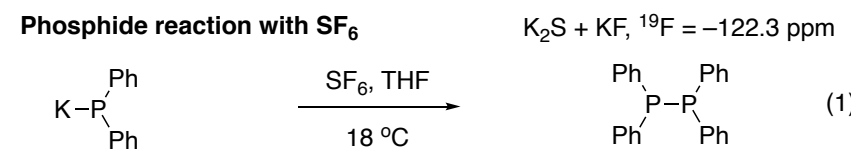

$6 a$

$7 a$

${ }^{31} \mathrm{P}=-10 \mathrm{ppm}$

${ }^{31} \mathrm{P}=-15.6 \mathrm{ppm}$

$95 \%$ yield ( $1 \mathrm{mmol}$ scale)

Sulfide detection:

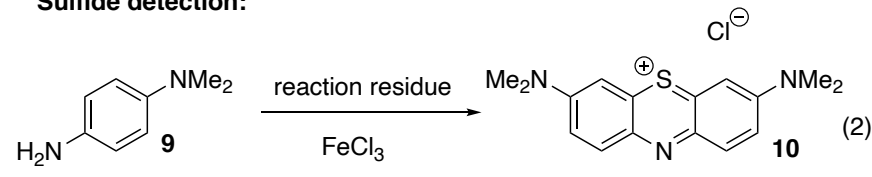

Lithium phosphide reaction with $\mathrm{SF}_{6}$
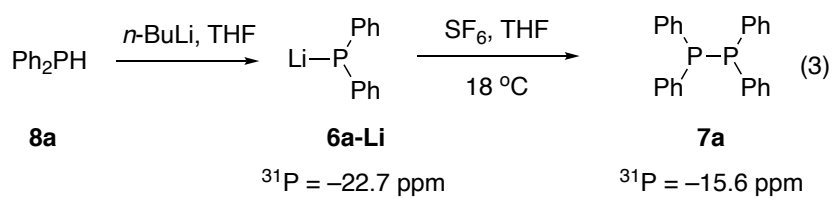

Cy= cyclohexyl

$\mathrm{Cy}_{2} \mathrm{PH} \stackrel{n \text {-BuLi, THF }}{\longrightarrow} \mathrm{Li}-\mathrm{P}_{\mathrm{Cy}}^{\mathrm{Cy}} \frac{\mathrm{SF}_{6}, \mathrm{THF}}{18^{\circ} \mathrm{C}}$

$8 b$

Low reactivity with $\mathrm{SF}_{6}$

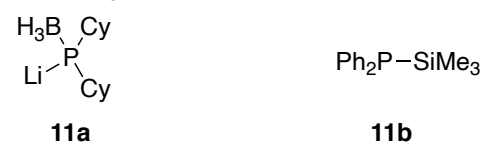

Scheme 2. Reaction of aryl and alkyl phosphides with $\mathbf{S F}_{6}$.

Complete consumption of the phosphide within seconds was again observed. Other metalloids were unreactive, phosphide-borane adduct 11a did not show appreciable reaction, ${ }^{21}$ and silyl-phosphine, $\mathrm{TMSPPh}_{2} 11$ was $^{2}$ also unreactive. These results indicate significant anionic character is necessary on the phosphorus centre for this reaction to proceed rapidly and cleanly.

Based on Dielmann's observation of nucleophilic attack by super-basic phosphines on $\mathrm{SF}_{6}$, we speculated anion $\mathbf{6 a}$ attacks $\mathrm{SF}_{6}$. While this would initially form fluoride 12a, which was not detected, electrophile 12a would be expected to immediately react with additional diphenylphosphide $\mathbf{6 a}$ to form $\mathbf{7 a}$ (Scheme 3, equation 1). Furthermore, compound $\mathbf{1 2 a}$ is not stable, and is known to disproportionate into 7a and phosphorane 13 (Scheme 3, equation 2). ${ }^{22}$ We did not observe $\mathbf{1 3}$ in the reaction mixture, suggesting that attack from diphenylphosphide was the faster route to form $\mathbf{1 2 a}$ though we could not rule out formation of $\mathbf{1 3}$ followed by reduction by $\mathbf{6 a}$. 
Proposed initial phosphide reaction with $\mathrm{SF}_{6}$ :

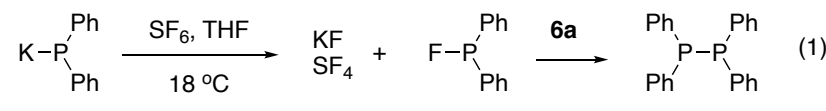

$$
\begin{aligned}
& 6 a \quad 12 a \quad 7 a
\end{aligned}
$$

Known disproportionation of fluorophosphines:

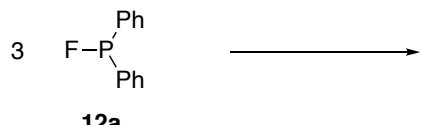

$12 a$

Kinetically stable fluorophosphines:

$$
\mathrm{Ph}^{\mathrm{Ph}-\mathrm{P}^{\prime}} \mathrm{Ph}
$$

$7 a$

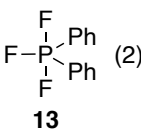

not observed

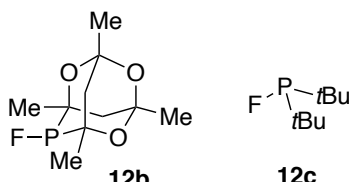

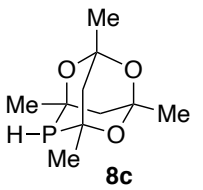

${ }^{31} \mathrm{P}=-50.6 \mathrm{ppm}$

${ }^{1} J_{\mathrm{P}-\mathrm{H}}=187 \mathrm{~Hz}$ no reaction with nBuLi, tBuLi, or $\mathrm{KH}$ in THF

Observation of fluorophosphines:

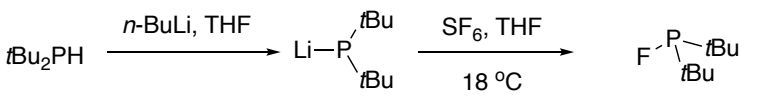

$$
\begin{aligned}
& \text { 8d } \\
& 6 c \\
& \text { 12c } \\
& { }^{31} \mathrm{P}=+40.9 \mathrm{ppm} \quad{ }^{31} \mathrm{P}=+212 \mathrm{ppm} \\
& 1 J_{\mathrm{P}-\mathrm{F}}=870 \mathrm{~Hz}
\end{aligned}
$$
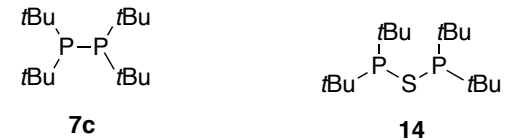

\section{Scheme 3. Further reactivity studies}

Lower sulfides of fluoride such as $\mathrm{SF}_{4}$ would also be expected to form, however these reactive species were not observed by ${ }^{19} \mathrm{~F}$ NMR spectroscopy, and we expect they were rapidly reduced under these conditions. In general, free lower fluorides of sulfur have not been observed in $\mathrm{SF}_{6}$ reduction reactions, with the exception of the pentafluorosulfanyl anion $\left(\mathrm{SF}_{5}-\right)$, which has been observed in Rueping's Hoge's, and Dielmann's systems.7,10,11 These systems all have counterions that impart kinetic stability to this anion, however instability of the pentafluorosulfanyl anion with simple cations such as potassium or lithium has been noted. ${ }^{23}$ We did not detect $\mathrm{SF}_{5}$ anion, and it is unlikely this fragile anion would survive the highly nucleophilic phosphides present in this reaction.

Detection of fluorophosphine intermediates would support this mechanistic hypothesis. In the reactions shown in Scheme 2, while the reaction of diphenylphosphides did not produce any compounds that exhibited coupling to ${ }^{19} \mathrm{~F}$ in the ${ }^{31} \mathrm{P}$ spectrum, traces of fluoride-containing phosphorus compounds were observed in the reaction that formed cyclohexyl compound 7b (Equation 4, Scheme 2). We speculated increasing the steric bulk of the phosphide would suppress dimerization. Increased steric bulk is also known to stabilize fluorophosphines. Unlike 12a, fluorophosphines 12b and $12 \mathrm{c}$ are known to be stable. ${ }^{24}$ We sought to generate the appropriate phosphides from the corresponding phosphines to see if $\mathbf{1 2} \mathbf{b}$ and $\mathbf{1 2 c}$ could then be prepared by treatment with $\mathrm{SF}_{6}$. Surprisingly, caged phosphine $\mathbf{8 c}$ was resistant to deprotonation by either $n \mathrm{BuLi}, t \mathrm{BuLi}$ or $\mathrm{KH}$ in THF over multiple hours, with the $\mathrm{P}-\mathrm{H}$ bond remaining intact as ascertained by proton-coupled NMR. ${ }^{25}$ Our attention turned to di-tert-butylphosphine $8 \mathrm{~d}$, which was deprotonated by $n \mathrm{BuLi}$ in THF to generate $6 \mathrm{cc}^{26}$ Upon exposure of phosphide fluorophosphine 12c was generated, and observed by 
${ }^{31} \mathrm{P}$ and ${ }^{19} \mathrm{~F}$ NMR spectroscopy, supporting our hypothesis that fluorophosphines are an intermediate formed in the reactions of phosphides with $\mathrm{SF}_{6} \cdot{ }^{27}$

The ability to stop at this intermediate, unlike previous examples was presumably due to the greater steric hinderance of the tert-butyl groups, however in addition to 12c, dimer 7c was still observed. Another prominent signal in the ${ }^{31 P}$ NMR spectrum at $+85.2 \mathrm{ppm}$ was observed, this closely matched that reported for compound 14 in an independent preparation. ${ }^{28}$ Compound $\mathbf{1 4}$ could be expected to form from either the reaction of sulfide ion with $\mathbf{1 2 c}$, or the reaction of sulfur with 7c followed by rearrangement.

In conclusion, these results represent a rare example of room-temperature reactivity of $\mathrm{SF}_{6}$. In contrast to some previously reported systems, the reaction proceeds without the need for ultraviolet or violet light irradiation. In addition, the reaction only takes seconds, and is to the best of our knowledge, the first example of a controlled reaction of a commercially available substance with $\mathrm{SF}_{6}$ at ambient temperature. While this reaction has potential as a rapid oxidative generation of diphosphines from the corresponding phosphides, its utility is limited by consideration of the high environmental burden of $\mathrm{SF}_{6}$, accordingly we did not conduct a large substrate scope of phosphides. Of greatest conceptual importance is the discovery of a new example of a highly accessible reaction that consumes the potent greenhouse gas $\mathrm{SF}_{6}$. While the relatively high reactivity of potassium diphenylphosphide would limit large scale application of this method for $\mathrm{SF}_{6}$ remediation, one potential application could be use of this technique to dispose of small amounts of $\mathrm{SF}_{6}$ residues from applications such as retinal surgery, ${ }^{29}$ or in field-servicing electrical equipment, ${ }^{2}$ that would otherwise be released into the atmosphere. In addition, the notable colour change of the diphenylphosphide reaction gives a good indication of when the reagent is exhausted.

Investigation of other phosphorus-based systems for remediation of $\mathrm{SF}_{6}$ that are capable of catalytic turnover with appropriate terminal reductants is underway and will be reported in due course.

"There are no conflicts of interest to declare".

Acknowledgements: Financial support from NSERC (Discovery Grant), and the Killam Foundation (B.S.N.H.) are acknowledged. Dr. Mike Lumsden and Mr. Xiao Feng (Dalhousie University) are thanked for assistance with NMR spectroscopy and massspectrometry respectively. Professors Laura Turculet and Mark Stradiotto (Dalhousie University) are thanked for providing compounds 6a, and 8a-8c. Professor Christopher Algar (Dalhousie University) is thanked for advice on detecting sulphide.

${ }^{1}$ M. Maiss, C. A. M. Brenninkmeijer, Environ. Sci. Technol., 1998, 32, 3077.

${ }^{2}$ L. G. Christophorou, J. K. Olthoff and R. J. van Brunt, IEEE Electr. Insul. Mag., 1997, 13, 20.

${ }^{3}$ Catalog of guidelines and standards for the handling and management of Sulfur Hexafluoride", U.S. Environmental Protection Agency Office of Air and Radiation Global Program Division (625 J) WASHINGTON DC 20460. https://www.epa.gov/sites/production/files/2016-02/documents/sf6utility7.pdf

${ }^{4}$ J. R. Case, F. Nyman, Nature, 1962, 193, 473.

${ }^{5}$ A) B. G. Harvey, A. M. Arif, A. Glöckner, R. D. Ernst, Organometallics, 2007, 26, 2872; B) P. Holze, B. Horn, C. Limberg, C. Matlachowski, S. Mebs, Angew. Chem., Int. Ed., 2014, 53, 2750.

${ }^{6}$ A) G. C. Demitras, A. G. MacDiarmid, Inorg. Chem., 1964, 3, 1198. B) L. Brewer, C.-A. Chang, B. King, Inorg. Chem, 1970, 9, 814.

${ }^{7}$ A) M. Rueping, P. Nikolaienko, Y. Lebedev, A. Adams, Green Chem., 2017, 19, 2571; B) J. A. Murphy, J. Garnier, S. R. Park, F. Schoenebeck, S.-z. Shou, A. T. Turner, Org. Lett., 2008, 10, 1227.

${ }^{8}$ G. lakobson, M. Pošta, P. Beier, J. Fluor. Chem., 2018, 213, 51.

${ }^{9}$ P. Tomar, T. Braun, E. Kemnitz, Chem. Commun., 2018, 54, 9753.

${ }^{10}$ R. F. Weitkamp, B. Neumann, H.-G. Stammler, B. Hoge, Chem. Eur. J., early view DOI: 10.1002/chem.202003504;

R. F. Weitkamp, B. Neumann, H.-G. Stammler, B. Hoge, Chem. Eur. J. earlyview DOI: 10.1002/chem.202005123

${ }^{11}$ F. Buß, C. Mück-Lichtenfeld, P. Mehlmann, F. Dielmann,

Angew. Chem., Int. Ed., 2018, 57, 4951.

12 L. Zámostná, T. Braun, Angew. Chem., Int. Ed., 2015, 54, 10652.

13 T. A. McTeague, T. F. Jamison, Angew. Chem., Int. Ed., 2016, 55, 15072. 
${ }^{14}$ S. Kim, Y. Khomutnyk, A. Bannykh, P. Nagorny, Org. Lett., 2021, 23, 190.

${ }^{15}$ A) D. Rombach, H-A. Wagenknecht, Angew. Chem., Int. Ed., 2020, 59, 300. B) D. Rombach, H.-A. Wagenknecht, ChemCatChem, 2018, 10, 2955.

${ }^{16}$ M. Xu, A. R. Jupp, Z.-W. Qu, D. W. Stephan, Angew. Chem, Int. Ed., 2018, 57, 11050.

${ }^{17} \mathrm{~A}$ video of the transformation is available in the Electronic Supplementary Information.

18 J. D. Cline, Limnol. Oceanogr., 1969, 14, 454.

${ }^{19}$ The phosphides are depicted as monomeric, unsolvated structures, but their solution behaviour may be more complex, see: I. J. Colquhoun, H. C. E. McFarlane, W. McFarlane, J. Chem. Soc., Chem. Commun., 1982, 220.

${ }^{20}$ R. A. Bartlett, M. M. Olmstead, P. P. Power, Inorg. Chem., 1986, 25, 1243.

${ }^{21}$ Small changes in the ${ }^{31} \mathrm{P}$ NMR spectra were observed after $\mathrm{SF}_{6}$ addition, but no dramatic changes like the alkali metal phosphide reactions.

${ }^{22}$ F. Seel, K. Rudolph, W. Gombler, Angew. Chem., Int. Ed. 1967, 6, 708

${ }^{23}$ K. Matsumoto, Y. Haruki, S. Sawada, S. Yamada, T. Konno, R. Hagiwara, Inorg. Chem., 2018, 57, 14882.

${ }^{24}$ N. Fey, M. Garland, J. P. Hopewell, C. L. McMullin, S. Mastroianni, A. G. Orpen, P. G. Pringle, Angew. Chem., Int. Ed., 2012, 51, 118.

${ }^{25}$ Only deprotonation of borane adducts of the cage have been reported. See: D. L. Dodds, Joelle Floure, M. Garland, M. F. Haddow, T. R. Leonard, C. L. McMullin, A. G. Orpen, P. G. Pringle, Dalton Trans., 2011, 40, 7137.

${ }^{26}$ R. A. Jones, A. L. Stuart, T. C. Wright, J. Am. Chem. Soc., 1983, 105, 7459.

${ }^{27}$ The products of this reaction were not isolated or separated. The literature ${ }^{31} \mathrm{P}$ shift and coupling constant for 12c, recorded in $\mathrm{CDCl}_{3}$, are $+214.9 \mathrm{ppm}\left(\mathrm{d},{ }^{1} J_{\mathrm{PF}}=865 \mathrm{~Hz}\right)$. See reference 24 .

${ }^{28}$ S. Yogendra, S. S. Chitnis, F. Hennersdorf, M. Bodensteiner, R. Fischer, N. Burford, J. J. Weigand, Inorg. Chem. $2016,55,1854$. Compound 14 is reported to have a ${ }^{31} \mathrm{P}$ chemical shift of $+86.6 \mathrm{ppm}$ in $\mathrm{CD}_{2} \mathrm{Cl}_{2}$ in this work.

${ }^{29} \mathrm{SF}_{6}$ bubbles are used to hold eye-structures in place after certain forms of eye surgery. See: U. Mester, P. Kroll, B. Völker, I. Kreissig, Ophthalmologica, 1983, 186, 151. 